H I G H L I G H T S

EVOLUTION

\section{A tangled web}

Earthworms, flies, fish and mammals share one important morphological feature - a segmented body plan. So, it seems logical that the common ancestor of the annelids, arthropods and vertebrates was also segmented. Until now, molecular data did not support this hypothesis, but recently published studies on the Central American wandering spider provide crucial evidence in its favour.

If segmentation originated only once, we would expect the genes that underlie this developmental process to be similar in the main animal groups. However, the mechanisms and genetic networks that initiate segmentation are different in Drosophila and vertebrates. In the fly, a transcriptional cascade causes progressive patterning along the anterior/posterior axis that results in formation of the embryonic segments. By contrast, vertebrate segments — somites — are formed one at a time through the expression of genes regulated by the segmentation clock. In the mouse, zebrafish and chicken, this process requires the activity of Notch and Delta, which are not needed for Drosophila segmentation.

However, Drosophila might not be a good model for arthropod segmentation mechanisms. Stollewerk et al. show that the spider Notch and Delta homologues are expressed in stripes during the segmentation process.

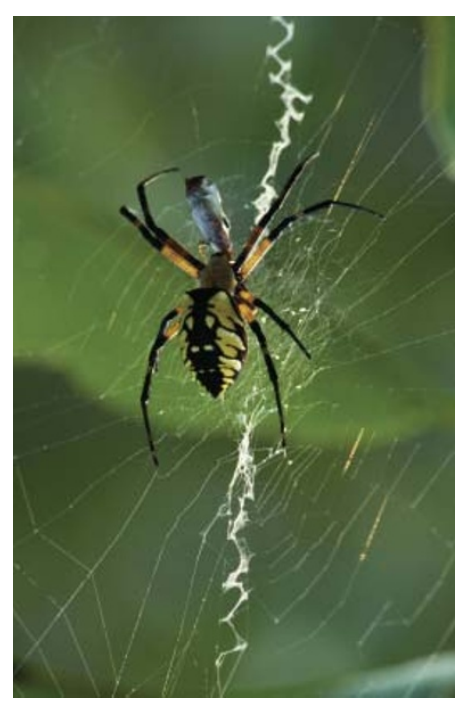

Moreover, it is clear from RNAi studies that these genes are necessary for correct segmentation in the spider, because embryos injected with double-stranded RNA targeted against Notch and Delta homologues showed aberrant segmentation.

So, segmentation mechanisms in some arthropods might be more similar to vertebrates than fly studies have indicated. This in turn implies that segmentation probably only originated once, in a common ancestor of the annelids, arthropods and vertebrates. Initially, this process probably depended on Notch and Delta. However, in the fly lineage, segmentation mechanisms diverged and became independent of this signalling pathway.

If you look hard enough, you can still find indications that the Notch/Delta signalling pathway was involved in segmentation in the ancestors of insects. In Drosophila, this pathway is involved in boundary formation in the eye, wing and legs. Furthermore, in grasshoppers as well as flies, there are genes that function in segmentation that are typically found downstream of the Notch/Delta signalling pathway. This is consistent with the idea that Notch/Delta once regulated these genes, but that their regulation has been replaced with other genes over evolutionary time in the insect lineage.

Thanks to this spider work, molecular and morphological data are no longer contradictory and both point to a single origin for the segmented body plan. The details of spider segmentation still need to be characterized and further studies will undoubtedly give an insight into how segmentation mechanisms have diverged in arthropods, vertebrates and annelids.

Catherine Baxter

(1) References and links ORIGINAL RESEARCH PAPER Stollewerk, A., Schoppmeier, M. \& Damen, W. G. M. Involvement of Notch and Delta genes in spider segmentation. Nature 423, 863-865 (2003)

WEB SITE

The Spider Project: $h t t p: / / w w w . g e n e t i k$. uni-koelin.de/groups/Damen/spider.html

\section{IN BRIEF}

\section{FUNCTIONAL GENOMICS}

A panoramic view of yeast noncoding RNA processing.

Peng, W. -T. et al. Cell 113, 919-933 (2003)

Cells are full of non-coding RNAs - in yeast they make up $95 \%$ of the nucleic-acid content. Yet the processing events that are involved in the maturation of these RNAs are not completely understood. Building on previous functional genomic and proteomic studies, the authors used microarray analysis to monitor the abundance and processing of noncoding RNAs in 468 mutant yeast strains. The results confirmed that many uncharacterized yeast proteins are involved in non-coding RNA biogenesis.

\section{DEVELOPMENTAL GENETICS}

Cell fate decisions within the mouse organizer are governed by graded Nodal signals.

Vincent, S. D. et al. Genes Dev. 17, 1646-1662 (2003)

Nodal, BMP and WNT regulate the patterning of the streak and the formation of midline-organizing tissues, but their specific contributions in different cell lines were poorly defined, until now. Using a conditional inactivation strategy, the authors show that removing Smad 2 affects the anterior definitive endoderm and precordal-plate progenitors, but not the primitive streak. They also show that Nodal activity in the posterior epiblast is required for the correct development of the anterior streak, and that both copies of Smad3 are required in Smad2-deficient mice to specify all axial midline tissues.

\section{HUMAN GENETICS}

Genome scan meta analysis of schizophrenia and bipolar disorder. Part I: methods and power analysis.

Levinson, D. F. et al. Am. J. Hum. Genet. 73, 17-34 (2003)

This is the first of three papers that discuss genome scan metaanalysis (GSMA) and its unbiased application to complex disorders. Levinson et al. examine the power of GSMA to determine linkage and identify the thresholds of significance for application to actual meta-analyses. In the same issue, Lewis et al. and Segurado et al. use GSMA to study schizophrenia and bipolar disorder.

\section{COMPUTATIONAL BIOLOGY}

\section{Computational discovery of internal micro-exons.}

Volfovsky, N. et al. Genome Res. 13, 1216-1221 (2003)

One area of annotating exon-intron structure that remains difficult is the identification of micro-exons - short exons of up to $25 \mathrm{bp}$ that are usually conserved between species and sometimes assist in alternative splicing events. Using their spliced alignment-correction procedure, the authors detected 224 previously unidentified micro-exons in four complete genomes - human, worm, fly and Arabidopsis. This new algorithm should help improve our understanding of genome variability and splicing machinery. 